Journal of Advanced Dielectrics

Vol. 2, No. 3 (2012) 1250016 (9 pages)

(C) World Scientific Publishing Company

DOI: $10.1142 /$ S2010135X12500166

\title{
HIGH DIELECTRIC CONSTANT COMPOSITE BASED ON CHLOROPHYLL A ENTRAPPED NANOPOROUS SILICA GEL
}

\author{
J. S. MANNA*, M. K. MITRA, S. MUKHERJEE and G. C. DAS \\ Department of Metallurgical and Material Engineering \\ School of Material Science and Nanotechnology \\ Jadavpur University, Kolkata 700032, India \\ *jhimlisarkar0@gmail.com
}

Received 25 February 2012

Revised 12 April 2012

Accepted 16 May 2012

Published 15 August 2012

\begin{abstract}
Chlorophyll a (naturally occurring Mg porphyrene) has been entrapped in nano/porous silica gel using sol-gel method at room temperature, producing a stable composite. HRTEM observation reveals regular nanoscale [around 15-20 nm diameter] distribution of aggregated polycrystalline chlorophyll a within porous silica matrix. UV-vis study also corroborates the presence of various aggregated chlorophyll a species within the system. Low field measurement shows almost 400 times enhancement of dielectric constant (1700) with incorporation of only $0.125 \mathrm{mg} / \mathrm{ml} \mathrm{of}$ chlorophyll and the loss is 0.5 at room temperature at $100 \mathrm{~Hz}$. The dielectric constant of the composite reaches 2500 as chlorophyll concentration becomes $1 \mathrm{mg} / \mathrm{ml}$. Observed strong space charge response to the external field and strong frequency dispersion of the dielectric properties of the composite can be attributed to the long-range electron delocalization [nomadic polarization] in chlorophyll a aggregates. The electric modulus $\left(\mathrm{M}^{*}\right)$ formalism used in this study enabled us to distinguish and separate various relaxation processes. It is found that with increasing chlorophyll concentration D.C. relaxation time decreases exponentially at room temperature. It is shown that observed relaxations do not perfectly follow the Debye response in high frequency region due to heterogeneous distribution of chlorophyll aggregates. The low values of room temperature activation energy calculated from Arrhenius plot reveal that polaronic hopping phenomena is absent at grain-interfacial region due to low thermal energy.
\end{abstract}

Keywords: Nanoporous silica gel; chlorophyll a; dielectric; dipole relaxation; activation energy.

\section{Introduction}

Organic-inorganic hybrids or nanocomposites are a new generation of advanced materials because they combine the advantages of both organic (e.g., flexibility, tunable dielectric permittivity, process ability) and inorganic components (e.g., rigidity, durability, thermal stability) ${ }^{1-4}$ Silica is considered as inorganic component for dielectric nanocomposites because of its lower dielectric constant and wide applications in electrical devices. Recently, a new class of multifunctional materials with unique combination of organic chromospheres in an

${ }^{*}$ Corresponding author. 
inorganic oxide glass network has been developed..$^{5,6}$ For that purpose sol-gel process offers the best method for incorporation of organic dyes, porphyrene $^{7}$ into inorganic porous gel matrices. To date, the study regarding porphyrenes in solid matrices especially in glassy matrix is very much limited due to the lack of suitable synthetic procedures. Among many organic chromophores, porphyrenes are attractive molecular dyes with flat and extended $\pi$ electron systems. ${ }^{8-10}$ Matthew Sauceda ${ }^{11}$ shows porphyrene exhibits high dielectric property as it has delocalized electrons around its structure which results in large dipole moment under external electric field. This can provide its potential use in the field of electronic devices. ${ }^{11}$ Chlorophyll $\mathrm{a}$ is an $\mathrm{Mg}$ porphyrene consisting of several conjugated nitrogen-containing rings surrounding a magnesium ion by coordinate covalent bonds. Venkatramaiaha et al., has incorporated tpp4 within glassy matrix and dielectric property has been investigated. ${ }^{12}$ There is hardly any data regarding the study of relaxation time and dielectric property of chlorophyll a incorporated silica gel in literature. The investigation of dielectric behavior of naturally occurring porphyrene-silica nanocomposite can be useful for various electronic applications. A chlorophyll-silica composite might offer a processable material with suitable properties retaining inherent dielectric characteristics of chlorophyll a. In our previous study we have isolated chlorophyll a and stabilized it within nanoporous silica gel matrix. The resulting composite is found to exhibit definite fluorescence characteristics and remain stable over a long period of time [above three months] without incorporating any stabilizing agent. ${ }^{13}$ This paper embodies the results of investigation of the dielectric behavior of such nanoporous silica chlorophyll composites with varying chlorophyll a concentration.

\section{Experimental}

The samples are prepared in two steps. Firstly, chlorophyll a is extracted from the fresh leaves and then the chlorophyll a pigments are entrapped into the silica gel matrix at room temperature keeping $\mathrm{pH}$ value at 6.8 with four different chlorophyll concentrations $[1,0.5,0.25$, and $0.125 \mathrm{mg} / \mathrm{ml}]$ denoted as concentration $4,3,2,1$ respectively in subsequent sections. Detailed synthesis procedure has been described in our previous publication. ${ }^{13}$
As prepared fine powder samples were pressed into pellets of $12 \mathrm{~mm}$ diameter and $2 \mathrm{~mm}$ thickness under the uniaxial pressure of 7 ton $/ \mathrm{cm}^{2}$ for $1 \mathrm{~min}$. Pellets were then coated with silver paste and dried in vacuum to establish ohmic contact. Measurements of different electrical parameters have been carried out using the Hioki LCR meter of type $3531 \mathrm{Z}$ Hi-Tester, Japan, with a frequency range $(42-5 \mathrm{MHz})$ having impedance accuracy ranging from $0.15 \%$ up to $4 \%$. Optical absorption spectra have been examined by Perkin Elmer, Lambda 35 uv-vis spectroscope. Structural characterization has been conducted using JEOL JEM-2100 HRTEM.

\section{Results and Discussion}

\subsection{UV-vis spectra}

The absorption spectra of silica doped with different concentrations of chlorophyll a are presented in Fig. 1. The observed spectrum is different from that of chlorophyll a in ethanol solvent [inset Fig. 1] as in ethanol, chlorophyll a exhibits two main characteristic bands at $665 \mathrm{~nm}$ known as $\mathrm{Q}$ band and one strong band near UV region at $433 \mathrm{~nm}$ called as Soret (B-band) band. However, all the chlorophyll a

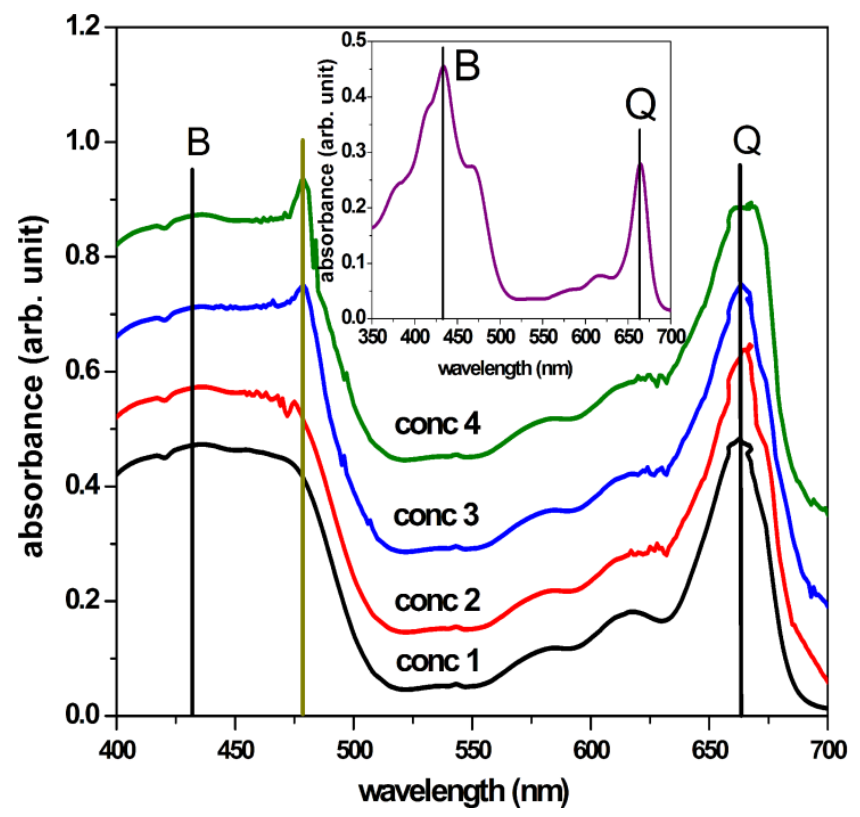

Fig. 1. (Color online) UV-vis absorption spectra of silica chlorophyll composites with different concentration of chlorophyll a. Inset shows the absorption spectra of chlorophyll a in ethanol solvent. 
doped silica samples show broadening of both absorption peaks around $665 \mathrm{~nm}$ and $433 \mathrm{~nm}$ along with appearance of new peak at $478 \mathrm{~nm}$. This indicates the presence of various aggregates in the silica matrix. Initially the broadening of peak is indicative of the presence of both $\mathrm{J}$ and $\mathrm{h}$ aggregates but at higher concentration more $\mathrm{J}$ aggregates are formed at the expense of $h$ aggregates. Thus the peak intensity at $478 \mathrm{~nm}$ increases. Increase in intensity of $478 \mathrm{~nm}$ peak with increasing chlorophyll concentration is a definite consequence of increasing amount of $\mathrm{J}$ aggregates. ${ }^{14}$ The red shift in the absorption spectrum of the aggregate relative to that of the monomer, due to excitonic coupling of the electronic transitions in the porphyrin $\pi$-systems. ${ }^{15}$ This phenomenon is consistent with an endon-end or slipped cofacial alignment of monomer transition dipoles. ${ }^{16}$ Thus in addition to interaction of pyrrole nitrogen with that of silica, ${ }^{13}$ chlorophyll molecules also interact with each other resulting in formation of various aggregates promoting heterogeneity. But as chlorophyll a concentration increases formation of $\mathrm{J}$ aggregates makes the composite more homogeneous.

\subsection{HRTEM observation}

Figure 2(a) shows the HRTEM images of bare amorphous silica. Images 2(b) and 2(c) show chlorophyll incorporated silica of concentration 4 . The aggregated porphyrene molecules represent domain of mobile charges. TEM images reveal these domains which are aggregated spherical chlorophyll a molecules with around $15-20 \mathrm{~nm}$ diameter. We suggest that the large dimension of aggregates may result from weakly intercalated porphyrin J-aggregates via van der Waals forces between alkyl chains, which is well consistent with a significant absorption spectral change. The inset of image 2(c) represent SAED pattern revealing polycrystallinity of aggregated chlorophyll a. This structure is uniformly dispersed in an insulating silica matrix representing a general nomadic form.

\subsection{Dielectric measurement}

From the dielectric response one can obtain, among others, important information on the relaxation processes occurring in the system. Different formalism has their own advantages, for example, a comparison of the permittivity and complex plane impedance presentation with electric modulus representation allows us to differentiate between local dielectric relaxation and long-range electrical conductivity. Material parameters have been calculated using following relations ${ }^{17}$ :

$$
\varepsilon^{\prime}=\frac{Z^{\prime \prime}}{\omega C_{0}\left(Z^{\prime 2}+Z^{\prime \prime 2}\right)},
$$

$$
\text { and } \quad \varepsilon^{\prime \prime}=\frac{Z^{\prime}}{\omega C_{0}\left(Z^{\prime 2}+Z^{\prime \prime 2}\right)} \text {, }
$$

where $C_{0}=\frac{\varepsilon_{0} A}{l}=$ capacitance of the sample cell $\left(\mathrm{A}=\right.$ sample area, $l=$ sample thickness, $\varepsilon_{0}=$ vacuum permittivity). $Z^{\prime}$ and $Z^{\prime \prime}$ are the real and imaginary part of the impedance, $\omega$ is the angular frequency of the applied field. The corresponding

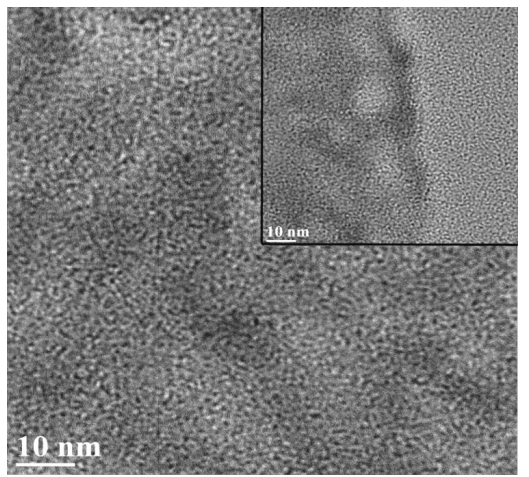

(a)

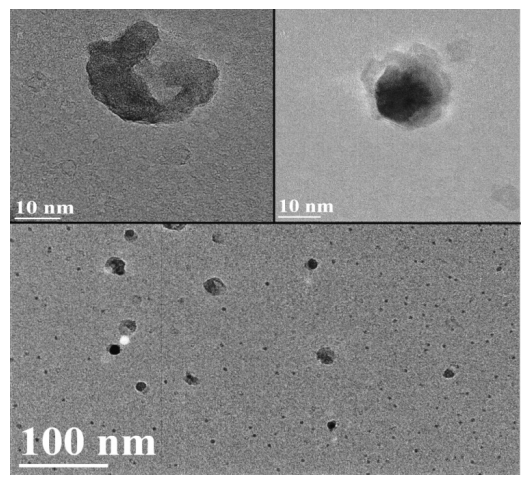

(b)

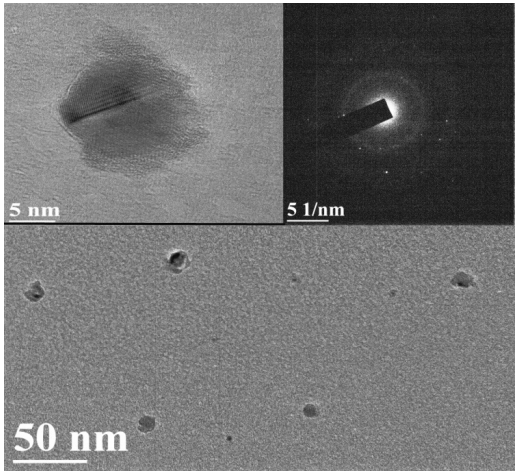

(c)

Fig. 2. (a) HRTEM image of bare silica gel. (b) HRTEM image of chlorophyll silica composite, showing polycrystalline chlorophyll agglomerates ranging from 2-20 nm. (c) HRTEM image of chlorophyll silica composite showing lattice and SAED pattern of polycrystalline composite. 
dielectric constant and dielectric loss are then:

$$
\begin{gathered}
\varepsilon=\varepsilon^{\prime}+i \varepsilon^{\prime \prime}=\frac{1}{i \omega C_{o} Z^{*}(\omega)}, \\
\tan \delta=\frac{Z^{\prime}}{Z^{\prime \prime}}=\frac{\varepsilon^{\prime \prime}}{\varepsilon^{\prime}}=\frac{M^{\prime \prime}}{M^{\prime}}
\end{gathered}
$$

Dependence of dielectric constant and dielectric loss as a function of frequency ranging from $100-10^{6} \mathrm{~Hz}$ at room temperature has been studied and plotted in Figs. 3 and 4 . In all the cases, the dielectric permittivity shows usual frequency dispersion behavior. Above $10^{4} \mathrm{~Hz}$, the dielectric permittivity value becomes nearly constant as shorter relaxation time in a high frequency field will prohibit degree of polarization in any material. ${ }^{18}$ Figure 3 inset shows the rise in permittivity values for all samples. This can be attributed to the incoherent dipole-dipole interaction in high frequency field due to the loss of charge carrier wave function coherence or phase information after each interaction event with molecular or crystal lattice vibrations. In a similar fashion, Fig. 4 shows the dielectric loss also decreases steeply as frequency increases without the presence of any loss peak within the observed frequency range. These results indicate that dielectric properties of the composites have strong frequency dispersion, which is a result of the space charge polarization mechanism in chlorophyll a aggregates. It can be observed that $\varepsilon^{\prime}$ as well as $\tan \delta$ are higher

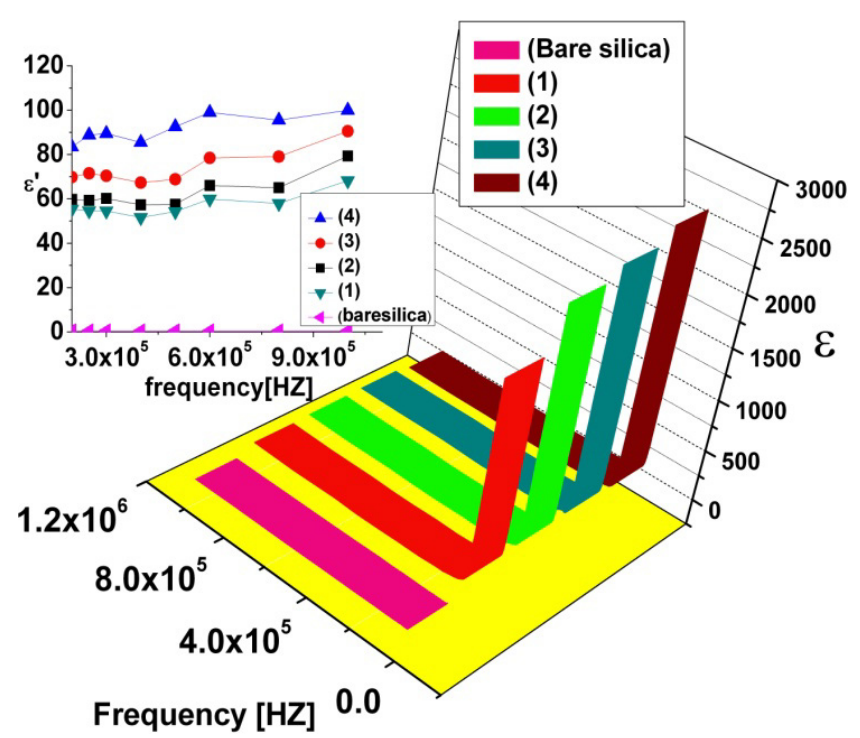

Fig. 3. (Color online) Frequency dispersion behavior of dielectric permittivity at room temperature for all samples. Inset shows the rise of $\varepsilon^{\prime}$ in high frequency region.

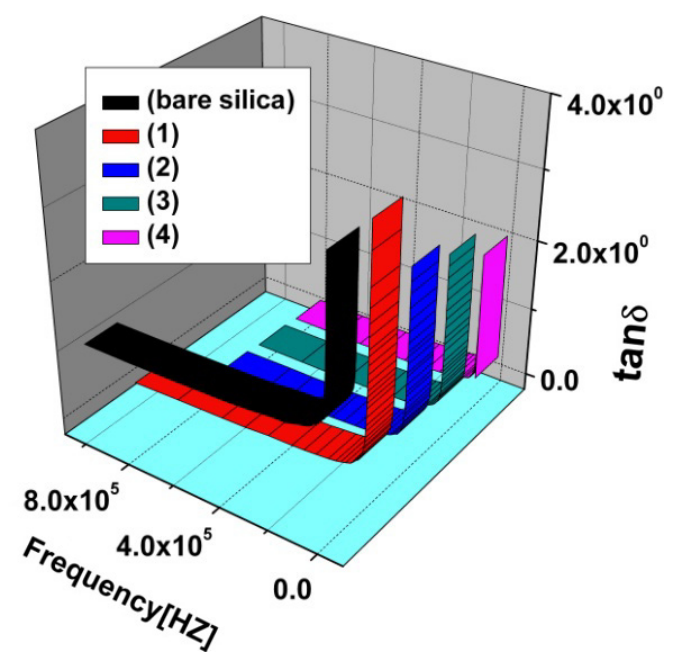

Fig. 4. (Color online) Dispersion of dielectric loss over the experimental frequency range.

for higher chlorophyll a concentrations. Chlorophyll molecules have delocalized $\pi$ electron over the porphyrene ring and hence dipole moment can easily be induced by applying electric field. At lower frequencies, the dipoles can respond in accordance to the field and space charge polarization is very high resulting in much higher dielectric permittivity and dielectric loss. At higher frequencies, dipole relaxation is much slower than the applied field frequency, so parameter values approach a minimum. Moreover, with increasing chlorophyll a concentration the dielectric permittivity also increases. It is attributed to the increase in number of charge carriers [contributed by delocalized $\pi$ of chlorophyll aggregates] within the silica matrix. This increase is due to the probability of charge buildup at the interfacial state between chlorophyll and silica host. ${ }^{17}$ The origin of the interfacial or Maxwell-Wagner-Sillars (MWS) relaxation is related to the existence of free charge carriers inside the system. Free charge carriers can migrate through the matrix under the influence of applied electrical field but get stacked at the interfaces between the media with significantly different resistivities. If the applied field is alternating, charges are forced to follow the motion of the field. A new migration of carriers occurs in the form of harmonic oscillating jumps between two potential wells ${ }^{19}$ along with a short-range diffusion of charges. ${ }^{20,21}$ Above phenomenon appears in heterogeneous media due to the accumulation of charges at the interfaces resulting in frequency-dependent permittivity. This interfacial state is expected to be a mixture of two 
materials with different conductivities and permittivities. $^{22}$ Figure 3 represents room temperature dielectric permittivity of bare silica and chlorophyll silica composite. At near $100 \mathrm{~Hz}$ the dielectric constant value increases with increased chlorophyll reinforcement in silica matrix. Composite with chlorophyll concentration 4 attains the highest $\varepsilon$ value of 2432. It can be explained in terms of the long-range electron orbital delocalization, also called nomadic polarization. Chlorophyll a has large

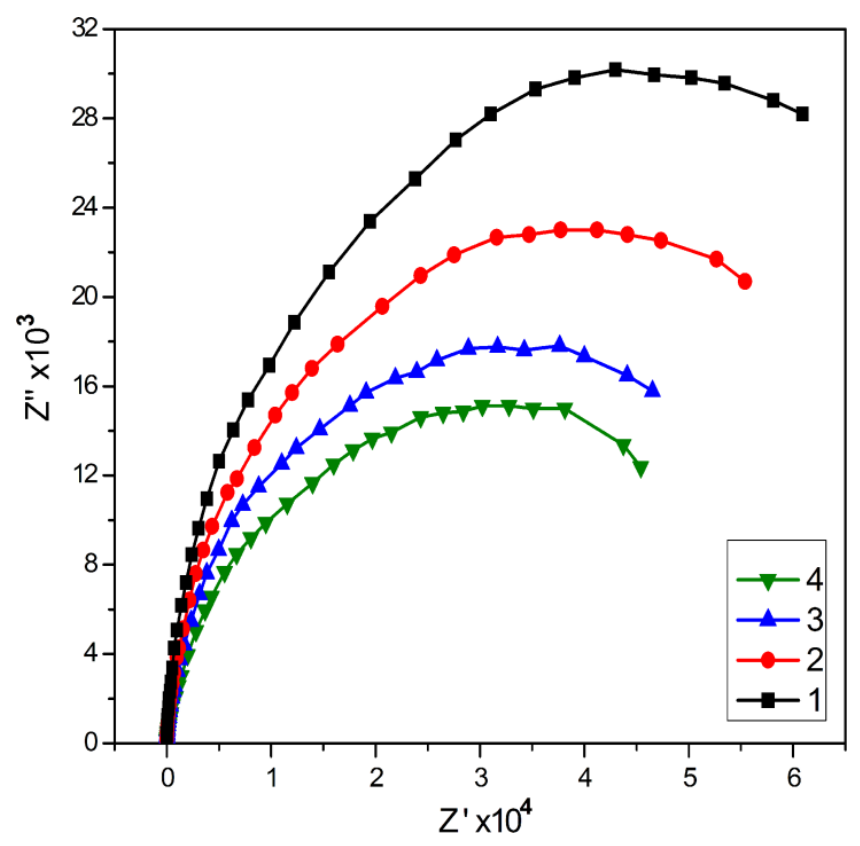

Fig. 5. (Color online) Complex impedance plane plot (Cole-Cole) of all the samples. planar structure. The $\pi$ electrons are completely delocalized over the entire molecule, and charges can move along suitable geometric long domains of chlorophyll aggregates. These structure represents domain for the mobile charges, as the concentration of the highly packed chlorophyll a molecules increases, the charge carrier concentration also increases resulting in increased permittivity. Figures 5 and 6 represent the complex plane diagram (Cole-Cole plot) in impedance and modulus representation respectively. The complex electric modulus $\left[M^{*}\right]$ is derived from the complex permittivity, according to the relationship defined by Macedo $e t a l .{ }^{21}$ The real and imaginary parts of the electric modulus $M^{\prime}$ and $M^{\prime \prime}$ can be calculated from $\varepsilon^{\prime}$ and $\varepsilon^{\prime \prime}$ as follows [21]:

$$
\begin{aligned}
M *(\omega) & =M^{\prime}(\omega)+\mathrm{j} M^{\prime \prime}(\omega)=\frac{1}{\varepsilon^{*}}, \\
M^{\prime}(\omega) & =\frac{\varepsilon^{\prime}(\omega)}{\left[\varepsilon^{\prime}(\omega)^{2}+\varepsilon^{\prime \prime}(\omega)^{2}\right]}, \\
M^{\prime \prime}(\omega) & =\frac{\varepsilon^{\prime \prime}(\omega)}{\left[\varepsilon^{\prime}(\omega)^{2}+\varepsilon^{\prime \prime}(\omega)^{2}\right]} .
\end{aligned}
$$

Interesting observation is that the impedance representation contains only one incomplete arc indicating single RC contribution. In contrast, the modulus representation shows superposition of two arcs; signaling two relaxation processes for every sample other than bare silica, the modulus representation of bare silica shows one broad arc (6a)). As we know, the measurement of electric modulus

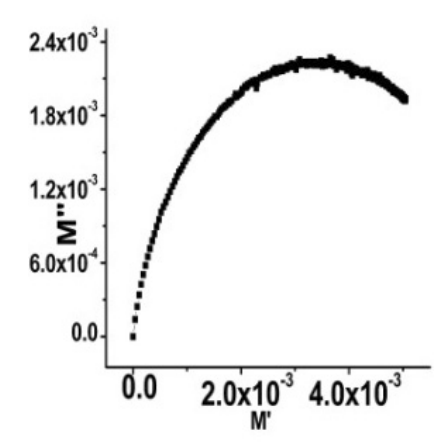

(a)

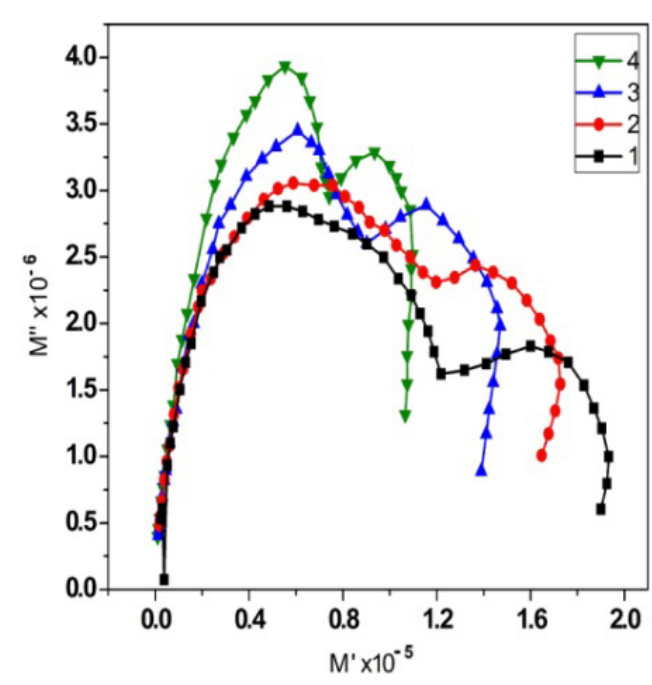

(b)

Fig. 6. (Color online) Cole-Cole plot in electrical modulus form, (a) bare silica and (b) chlorophyll silica composites. 
$M=i \omega C_{0} Z$ is a useful function to eliminate electrode effect. This approach gains popularity when the reciprocal complex permittivity was discussed as an electrical analogue to the mechanical shear modulus. ${ }^{23}$ Physically, the electrical modulus is the relaxation of the electric field in the material when the electric displacement remains constant. The modulus therefore represents the real dielectric relaxation process. This representation in the analysis of relaxation properties already finds usefulness for vitreous ionic conductors ${ }^{24}$ and polycrystalline ceramics. ${ }^{25}$ In case of impedance representation, surface polarization plays an important role to overshadow the interfacial contribution produced by the inorganic-organic interfaces. High frequency side shows the grain contribution and low frequency side represents the grain boundary contributions. Grain and grain boundary resistance, capacitance, and corresponding relaxation can be evaluated deploying the following equations:

$$
\begin{gathered}
{\left[Z^{\prime}-\left(\frac{R_{G}+R_{G B}}{2}\right)\right]^{2}+\left[Z^{\prime \prime}\right]^{2}} \\
=\left[\frac{R_{G}+R_{G B}}{2}\right]^{2} \\
{\left[M^{\prime}-C_{0}\left(\frac{1}{C_{G}}+\frac{1}{C_{G B}}\right)\right]^{2}+\left[M^{\prime \prime}\right]^{2}} \\
=\left[\frac{C_{0}}{C_{G}}+\frac{C_{0}}{C_{G B}}\right]^{2}
\end{gathered}
$$

Modulus representation (6b) clearly reflects that as chlorophyll a concentration increases, grain boundary contribution decreases and grain capacitance increases. A kinetic investigation by Julio C. de Paula et al. $[1995]^{16}$ confirmed that the aggregation process of chlorophyll a is cooperative involving three steps:

(1) formation of a nucleating species, probably a dimer of chlorophylls;

(2) formation of large aggregates with little or no secondary structure; and

(3) conformational change to form structured aggregates.

Thus monomeric concentration influence the structure of chlorophyll aggregate. At lower concentration, dimers or small oligomers of chlorophyll with less amount of folded aggregates are found. As the concentration increases secondary structure [e.g., helical or spherical] predominant from these small aggregates. Hence increased concentration induces homogeneity of secondary structure within matrix. As a consequence the grain capacitance increases. Capacitance values associated with the two $R C$ elements are different as peak heights corresponding to grain and grain boundary region are different. Relaxation time of the oscillating dipoles situated at grain and grain boundaries can be estimated from the frequency value $\left(\nu_{m}\right)$ of the high and low frequency $M^{\prime \prime}$ peak using the relation: $\omega_{m} \tau=1$. Close values of the two peak frequencies also indicate that the dielectric behavior is due to the intrinsic electrical characteristics of the sample. High frequency grain peaks and low frequency grain boundary peaks evidently move toward each other as the chlorophyll a concentration increases inside the nanoporous silica-gel matrix, due to the fact that at lower chlorophyll concentrations presence of various chlorophyll species e.g., dimers, oligomer,

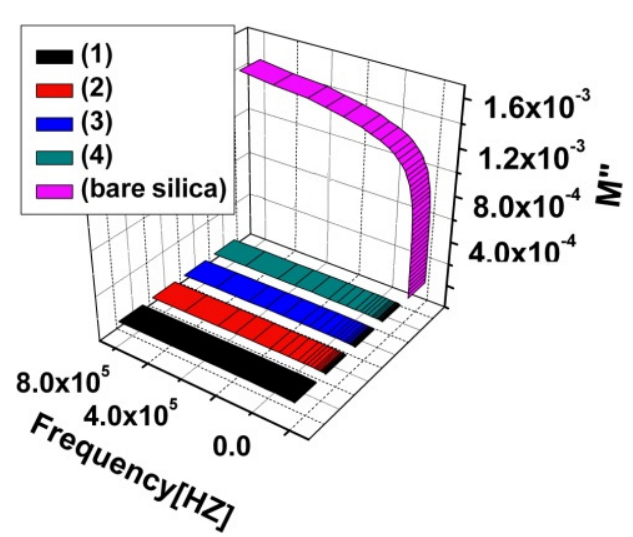

(a)

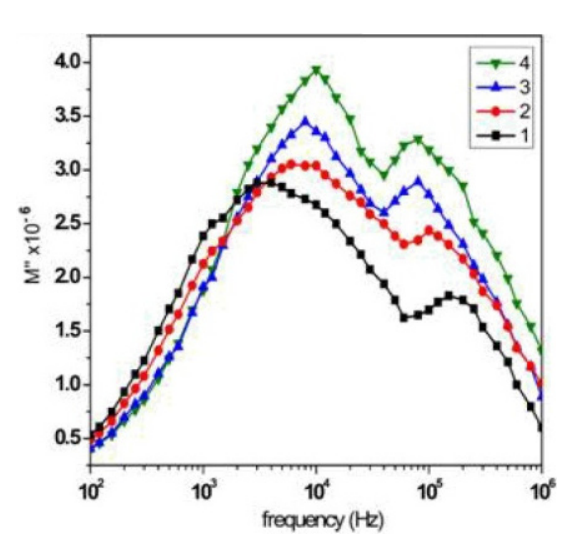

(b)

Fig. 7. (Color online) $M^{\prime \prime}$ versus $\log f$ curve of (a) all samples including bare silica and (b) chloro-silica composites. 


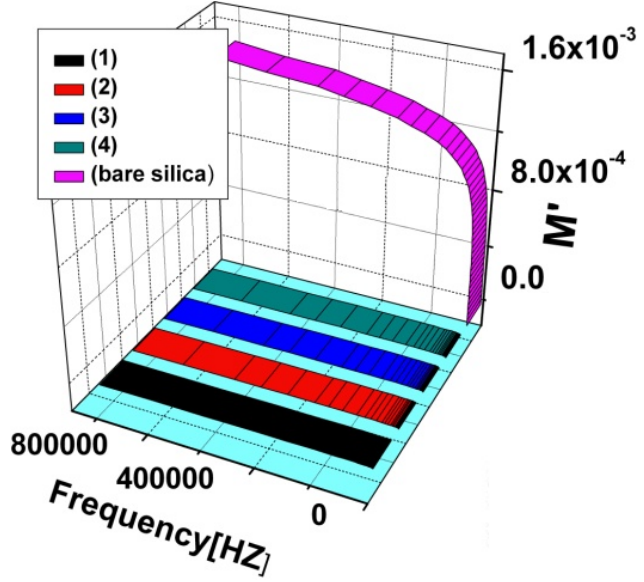

(a)

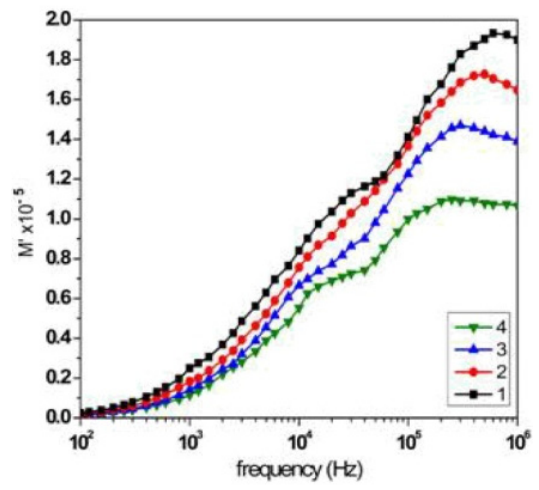

(b)

Fig. 8. (Color online) $M^{\prime}$ versus $\log f$ curve of (a) all samples and (b) with different chlorophyll concentrations.

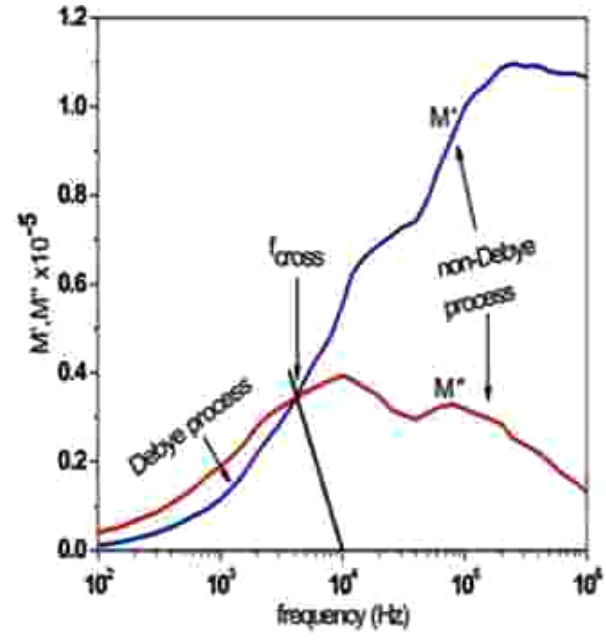

(a)

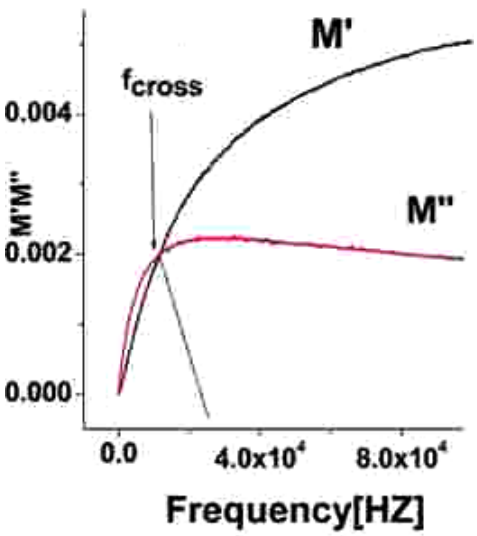

(b)

Fig. 9. (Color online) $M^{\prime}(\omega)$ and $M^{\prime \prime}(\omega)$, obtained at room temperature for conc. 1 and bare silica.

and less amount of secondary structure lead to more heterogenesity of the system. At increased concentration, formation of secondary structure makes the system more homogeneous, hence the two peaks come closer. So, for much higher chlorophyll a concentration these two peaks are supposed to coincide to produce an intermediate characteristic relaxation time for effective homogeneity. But up to the highest concentration studied, heterogeneity is preserved.

In Fig. 7(a) for all samples including bare silica, 7(b) for composites with different chlorophyll concentration, an additional process can be distinguished in the low frequency range, below $1 \mathrm{kHz}$. It is about one order of magnitude lower than the above-mentioned grain boundary dipole relaxation time $\left(\tau_{\mathrm{GB}}\right)$. The nature of the plot at high frequency region imply that it does not follow perfect Debye response like polymer filler composite and shows an asymmetric distribution. Since the amplitudes of the two peaks vary significantly, we plotted further $M^{\prime}(\omega)$ and $M^{\prime \prime}(\omega)$, obtained at room temperature, so that the details could be seen better (Fig. 9).

Figure 8 represents the real part of modulus with increasing frequency of all samples (a) and composites with different chlorophyll concentrations (b). The increase in the values of $M^{\prime}$ and the subsequent development of $M^{\prime \prime}$ peaks with increasing frequency is a characteristic behavior of a dielectric dispersion. The constant value of $M^{\prime}$ at higher frequencies is 


\section{J. S. Manna et al.}

due to the fact that interfacial polarization is inactive at higher frequencies, as large dipoles developed at the interfaces cannot follow the electric field when the frequency is high. The decrease in the values of $M^{\prime \prime}$ with increase in the chlorophyll content, observed, is due to exactly a result of the logarithmic law of mixtures. ${ }^{22}$

The low frequency process can be easily distinguished from Fig. 9 [(a) bare siliica, (b) silica chlorophyll composite conc. 1] where $M^{\prime}$ and $M^{\prime \prime}$ are plotted simultaneously with respect to frequency. It is believed that this low frequency relaxation process discussed above originates from the dc conductivity of a heterogeneous system. ${ }^{26-28}$ This behavior can be ascribed to Debye-like formula in the following modulus representation:

$$
M_{\mathrm{dc}}^{\prime \prime}(\omega)=M_{\infty} \frac{\omega \tau_{\mathrm{dc}}}{1+\left(\omega \tau_{\mathrm{dc}}\right)^{2}} .
$$

Assuming that the low frequency relaxation process follows simple Debye dispersion, its characteristic relaxation time from the fitting procedure can be evaluated as schematically shown in Fig. 9. The crossing frequency $\left(\nu_{\text {cross }}\right)$ as depicted in Fig. 9 can be considered as peak frequency $\left(\nu_{\max }\right)$ for calculation of dc relaxation time $\left(\tau_{\mathrm{dc}}\right){ }^{29}$

Now, for calculation of grain and interfacial activation energy, we can consider well known Arrhenius formalism:

$$
\tau=\tau_{0} \exp \left(\frac{-E}{k_{B} T}\right)
$$

If we consider the value of $\tau_{\text {dc }}$ to be equal to $\tau_{0}$, we can easily prescribe the nature of transport phenomena taking place inside the heterostructure by noticing the value of activation energies at room temperature. Numerical values of different relaxation time along with grain and interfacial activation energies for all samples are tabulated in
Table 1. As expected the amorphous bare silica exhibits only single relaxation time with $\tau_{\mathrm{dc}} 8.98 \mathrm{E}-5$. As the heterogeneity of the system increases in conc. 1 from bare silica the activation energy suddenly increases from bare silica. With the chlorophyll a concentration $\tau_{\mathrm{dc}}$ maintains a simple exponential dependence and gradually decreases on the higher concentration side due to the abovementioned homogeneity of the system. It is exhibited in Fig. 10 along with the inset showing the simultaneous variation of grain $\left(E_{G}\right)$ and interfacial $\left(E_{\mathrm{GB}}\right)$ activation energy with different chlorophyll content. The bare silica has an activation energy $0.017 \mathrm{eV}$ at room temperature. Low values of both $E_{G}$ and $E_{\mathrm{GB}}$ suggest that polaronic hopping phenomena is absent at grain-interfacial region due to low thermal energy. As the porphyrene incorporation increases carrier concentrations, $E_{G}$ and $E_{\mathrm{GB}}$ decays exponentially even at constant temperature.

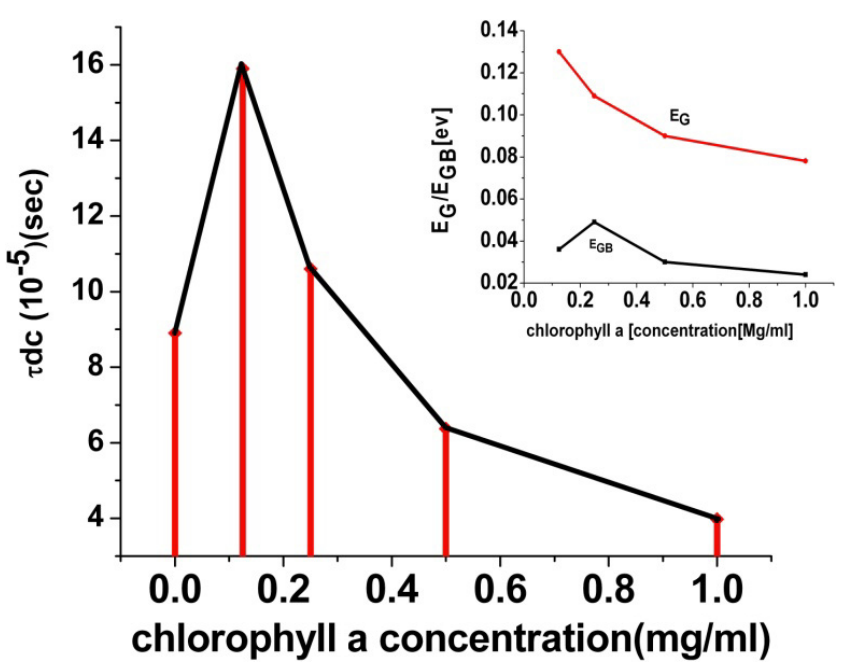

Fig. 10. (Color online) Dependence of $\tau_{\text {dc }}$ with the chlorophyll a concentration. Inset shows the simultaneous variation of $\left(E_{G}\right)$ and $\left(E_{\mathrm{GB}}\right)$ activation energy with different chlorophyll content.

Table 1. Experimental values of calculated relaxation time corresponding to grain and interfacial dipoles and of dc conductivity along with grain and interfacial activation energy for all samples.

\begin{tabular}{cccccc}
\hline Chlorophyll concentration & $\tau_{\mathrm{GB}}(\mathrm{sec})$ & $\tau_{G}(\mathrm{sec})$ & $\tau_{\mathrm{dc}}(\mathrm{sec})$ & $E_{\mathrm{GB}}(\mathrm{eV})$ & $E_{G}(\mathrm{eV})$ \\
\hline $0.125 \mathrm{mg} / \mathrm{ml}$ & $3.98 \times 10^{-5}$ & $1.06 \times 10^{-6}$ & $1.59 \times 10^{-4}$ & 0.036 & 0.130 \\
$0.25 \mathrm{mg} / \mathrm{ml}$ & $1.59 \times 10^{-5}$ & $1.59 \times 10^{-6}$ & $1.06 \times 10^{-4}$ & 0.049 & 0.109 \\
$0.5 \mathrm{mg} / \mathrm{ml}$ & $1.99 \times 10^{-5}$ & $1.99 \times 10^{-6}$ & $6.37 \times 10^{-5}$ & 0.030 & 0.090 \\
$1 \mathrm{mg} / \mathrm{ml}$ & $1.59 \times 10^{-5}$ & $1.99 \times 10^{-6}$ & $3.98 \times 10^{-5}$ & 0.024 & 0.078 \\
Bare silica & $\tau(\mathrm{sec}) 4.47 \times 10^{-5}$ & $8.97 \times 10^{-5}$ & $\mathrm{E}(\mathrm{eV}) 0.017$ \\
\hline
\end{tabular}




\section{Conclusions}

The local dielectric relaxation in silica chlorophyll composites has been studied by employing electric modulus formalism. Interfacial or MWS relaxation is evident in the dielectric spectra of the examined systems due to the interfaces introduced by the chlorophyll a aggregated molecules. The observed relaxations do not perfectly follow the Debye response in high frequency region for the distribution of relaxation times like various polymer filler composites. Assuming the low frequency relaxation process follows simple Debye dispersion, the dc relaxation time has been calculated. The calculated low activation energy from Arrhenius equation shows that polaronic hopping phenomena is absent at graininterfacial region due to low thermal energy. The dielectric constant value of the composites can reach up to 2432 at room temperature and $100 \mathrm{~Hz}$ from bare silica \{dielectric constant around 2 \}, when the concentration of chlorophyll a in the composite is $1 \mathrm{mg} /$ $\mathrm{ml}$. Because of the long-range electron orbital delocalization, also called nomadic polarization in chlorophyll a aggregates. Grain capacitance also increases as a result of increased homogeneity of the composite with increased chlorophyll concentration. These information regarding electrical characterization of the composite may be useful for the organic capacitor fabrication.

\section{Acknowledgments}

One of our authors (J. S. Manna) wishes to thank the Council for Scientific and Industrial Research [INDIA] for providing financial support during the execution of the research work. Authors acknowledge Mr. Suman Nandi for the dielectric measurements.

\section{References}

1. Y. Chen and O. I. Jude, Chem. Mater. 11, 1218 (1999).

2. C. Guizard, A. Bac, M. Barboiu and N. Hovnanian, Sep. Purif. Technol. 25, 167 (2001).

3. C. J. T. Landry, B. K. Coltrain, J. A. Wesson and N. Zumbulyadis, Polymer 33, 1496 (1992).

4. B. M. Novak, D. Auerbach and C. Verrier, Chem. Mater. 6, 282 (1994).

5. M. Pedziwiatr, R. Wiglusz, A. Graczyk and J. Legendziewicz, J. Alloys Compd. 451, 46 (2008).
6. L. Wei, Q. Chen and Y. Gu, J. Alloys Compd. 501, 313 (2010).

7. D. Dolphin, The Porphyrins, Vol. 3: Physical Chemistry Part A (Academic Press, New York, 1978).

8. K. Smith, Porphyrins and Metalloporphyrins (Elsevier, Amsterdam, 1976).

9. P. N. Prasad and D. J. William, Introduction to Nonlinear Optical Effects in Molecules and Polymers (Wiley, New York, 1991).

10. M. L. Curri, A. Petrella, M. Striccoli, P. D. Cozzoli, P. Cosmaa, A. Agostiano, Synthetic Met. 139, 593 (2003).

11. http://altair.physics.ncsu.edu/research.htm.

12. N. Venkatramaiah, N. Veeraiah and R. Venkatesana, J. Alloys Compd. 509, 2797 (2011).

13. J. S. Manna, S. Basu, M. K. Mitra, S. Mukherjee and G. C. Das, J. Mater. Sci. 20, 1068 (2009).

14. I. M. Hodge and C. A. Angell, J. Chem. Phys. 67(4), 1647 (1977).

15. M. Adachi, M. Yoneyama and S. Nakamura, Langmuir 8, 2240 (1992).

16. J. C. de Paula, J. H. Robblee and R. F. Pastermack, Biophys. J. 68, 335 (1995).

17. S. Nandy, S. Mallick, P. K. Ghosh, G. C. Das, S. Mukherjee, M. K. Mitra and K. K. Chattopadhyay, J. Alloys Compd. 453, 1 (2008).

18. I. M. Hodge and C. A. Angell, J. Chem. Phys. 67(4), 1647 (1977).

19. S. Wang, Z. Ahmad and J. E. Mark, Chem. Mater. 6, 943 (1994).

20. H. Xu, Y. Bai, V. Bharti and Z. Y. Cheng, J. Appl. Polym. Sci. 82, 70 (2001).

21. G. M. Tsangaris, G. C. Psarras and A. J. Kontopoulos, J. Non-Cryst. Solids 1164, 131 (1991).

22. S. A. Chen and C. S. Liao, Macromolecules 26, 2810 (1993).

23. N. G. McCrum, B. E. Read and G. Williams, A Inelastic and Dielectric Effects in Polymeric Solids (Wiley, New York, 1967).

24. P. B. Macedo, C. T. Moynihan and R. A. Bose, Phys. Chem. Glasses 13, 171 (1972).

25. J. Liu, C.-G. Duan, W.-G. Yin, W. N. Mei, R. W. Smith and J. R. Hardy, Chem. Phys. 119, 2812 (2003).

26. A. K. Jonscher, Dielectric Relaxation in Solids (Dielectrics Press, London, 1983).

27. A. K. Jonscher, Universal Relaxation Law (Dielectrics Press, Chelsea, London, 1996).

28. R. Coelho, Physics of Dielectrics for the Engineer, Chapter 8 (Elsevier, Amsterdam, 1979).

29. A. Molak, M. Paluch, S. Pawlus, J. Klimontko, Z. Ujma and I. Gruszka, J. Phys. D: Appl. Phys. 38, 1450 (2005). 